\title{
Integrated Location-Production-Distribution Planning in a Multiproducts Supply Chain Network Design Model
}

\author{
Vincent F. Yu, ${ }^{1}$ Nur Mayke Eka Normasari, ${ }^{1}$ and Huynh Trung Luong ${ }^{2}$ \\ ${ }^{1}$ Department of Industrial Management, National Taiwan University of Science and Technology, No. 43, Section 4, \\ Keelung Road, Taipei 10607, Taiwan \\ ${ }^{2}$ Industrial and Manufacturing Engineering, Asian Institute of Technology, 58 Moo 9, Paholyothin Highway Klong Luang, \\ Pathumthani 12120, Thailand
}

Correspondence should be addressed to Nur Mayke Eka Normasari; mayke_sari@yahoo.com

Received 31 October 2014; Revised 7 February 2015; Accepted 23 February 2015

Academic Editor: Xuefeng Chen

Copyright (C) 2015 Vincent F. Yu et al. This is an open access article distributed under the Creative Commons Attribution License, which permits unrestricted use, distribution, and reproduction in any medium, provided the original work is properly cited.

\begin{abstract}
This paper proposes integrated location, production, and distribution planning for the supply chain network design which focuses on selecting the appropriate locations to build a new plant and distribution center while deciding the production and distribution of the product. We examine a multiechelon supply chain that includes suppliers, plants, and distribution centers and develop a mathematical model that aims at minimizing the total cost of the supply chain. In particular, the mathematical model considers the decision of how many plants and distribution centers to open and where to open them, as well as the allocation in each echelon. The LINGO software is used to solve the model for some problem cases. The study conducts various numerical experiments to illustrate the applicability of the developed model. Results show that, in small and medium size of problem, the optimal solution can be found using this solver. Sensitivity analysis is also conducted and shows that customer demand parameter has the greatest impact on the optimal solution.
\end{abstract}

\section{Introduction}

A supply chain is a network that consists of a set of geographical facilities (suppliers, plants, and warehouses or distribution center). Through those facilities, there is material flow from supplier, plant, warehouse, and end in the customer. It aims at bringing the right amount of the right product to the right place at the right time [1]. Moreover, a supply chain network design is a strategic decision that has high risk and long-term impact in the supply chain system. The impact of efficiency supply chain has become more important on the business competitiveness [2]. The topic has triggered both researchers and practitioners to pay more attention to the supply chain network design. Many studies have been conducted to help the practitioner in making the best decision on a supply chain network. Indeed, determining the best supply chain network is a challenge, starting with problem identification, problem formulation, and its final solution and decision.

Today's competition among companies and market's globalization have resulted in firms developing a supply chain that can respond quickly to customers' need. In the current business environment, a company has to reduce costs while improving its customer service level to remain competitive [3], which also helps maintain profit margins. In order to achieve these goals, a company should appropriately select the location of the factory and the distribution center [46]. According to Altiparmak et al. [7], an optimal, efficient, and effective supply chain platform is provided by supply chain network (SCN) design, which also helps to improve supply chain performance. Moreover, Ballou [8] noted that the SCN design goal is to maximize the financial ratio, which is relevant to the objective of gaining the maximum return of investment at the minimum cost.

Supply chain management is divided into two levels: strategic and operational. The strategic level primarily is about the cost-effective location of facilities (plants and distribution centers), the flow of products throughout the entire supply chain system, and the assignment in each echelon [9-12]. The operational level is about the safety stock of each product in each facility, the replenishment size, frequency, 
transportation, and lead time, and the customer service level. According to Beamon [13], determining an effective supply chain is an important component in supply chain design. In addition, the decisions regarding in which facilities the product should be made and how to serve customers are very critical [14].

This paper provides a system optimization perspective in strategic planning for a supply chain network design that allows simultaneously determining the best location of facilities, raw material flow, and product flow on various echelons. Previous research on strategic planning for supply chain starts by considering the basic problems that have several characteristic, namely, single-period, single-product, single-echelon, and deterministic [15-25]. However, this is not sufficient to cope with the realistic problem. Therefore, many extensions to the basic problem are needed to make the problem more realistic. In this case, our paper considers multiperiod, multiproduct, and multiechelon which are still in deterministic situation to make the basic strategic planning problem more reasonable. A supply chain network design model helps managers conduct strategic planning for their company by selecting the best facility location that minimizes the total cost of the supply chain. The proposed multiproduct supply chain network design model herein helps in choosing the appropriate location of a new plant and distribution center as well as the distribution of the product and raw materials when the demand varies during the different time period. Moreover, multiechelon which represents the multitype of facility is the crucial aspect to be considered in strategic planning.

The paper is organized as follows. Section 2 presents previous research to find the gap between this study and earlier related research. Section 3 describes the problem definition and the proposed mathematical model. Section 4 contains the numerical experiments for the small and medium cases. Section 5 offers a sensitivity analysis result of the proposed model. Finally, Section 6 consists of the conclusion and suggestions for future research.

\section{Literature Review}

Several research integrated supply chain network designs have been developed to help practitioners solve their supply chain planning. Syarif et al. [26] studied a multiechelon, single-product logistic chain network model and proposed a novel technique as the solution method, called the spanning tree-based genetic algorithm (st-GA). The model is formulated by using a mixed integer liner programming (MILP) model. Their model only considers a single-product. To demonstrate the effectiveness and efficiency of their proposed method, it is compared to the traditional matrix-based genetic algorithm (m-GA). The experiment result shows that the proposed method presents a better solution almost all time and also performs better in computational time and memory for computation.

Jakeman et al. [27] considered the strategic and operational planning level decision in their research by developing a static model for a multiechelon, multiproduct supply chain network design. They examined the single source distribution system. For their solution, they used Lagrangian relaxation and a heuristic algorithm that utilizes the Lagrangian solution. The result of their computation shows that the solution method is both efficient and effective.

Shen [20] proposed a supply chain network design model with profit maximization as the objective function, but it considers only a single-product. In addition, the company may lose the customer if the product's price is higher than the customer reserve price. Altiparmak et al. [28] studied a single-product, multiechelon, and multiobjective SCN design. They set up a solution procedure based on the genetic algorithm (GA) to find the optimal solution to their problem. The multiobjective optimization problem consists of many optimal solutions, called Pareto-optimal solutions. The problem is formulated as a multiobjective mixed integer nonlinear programming model. The objectives are to minimize total cost, maximize customer service, and maximize utilization of the distribution centers (DCs).

Altiparmak et al. [7] presented a solution procedure for a multiproduct supply chain network (SCN) design based on the steady-state genetic algorithm (ssGA) with a new encoding structure. They considered a single source, multiproduct, and multiechelon supply chain network design in which the number of customers and their demands are assumed to be known. The problem, which is the NP-hard problem, is provided in mixed integer programming formulation. In order to investigate the effectiveness of the ssGA, three other heuristic approaches are also used: Lagrangian heuristic (LH), hybrid genetic algorithm (hGA), and simulated annealing. The experiment's results show that ssGA has a better solution than the other heuristic approaches used. Ying-Hua [29] adopts the model developed by Altiparmak et al. [7], which considers a single source, multiproduct, and multiechelon supply chain network design, but the model only has multisources instead of a single source. Additionally, the plants and DCs that are open are known. To verify the efficiency of his proposed method, he compared it to other algorithms, such as mathematical programming, the simple genetic algorithm, the coevolutionary genetic algorithm, and the constraint-satisfaction genetic algorithm. The experimental result in Taiwan's textile industry shows that the proposed method of Ying-Hua [29] performs better than other researchers' methods.

Bhutta et al. [30] developed an integrated location, production, distribution, and investment mixed integer linear programming (MILP) model in a two-echelon, multiproduct, multiperiod, and flexible facility capacitated with maximum profit as the objective function. Cóccola et al. [31] set up an integrated production and distribution MILP model in a multiechelon, multiproduct, and single-period setting with minimum total cost as the objective function. They conducted an empirical numerical experiment on six European countries. Fahimnia et al. [32] presented an integrated production and distribution planning MILP model for a two-echelon SC that considers several real world variables and constraints. They used GA to optimize the model and solved the medium-size case problem in their numerical experiment. In addition, Bashiri et al. [33] and Badri et al. [34] developed a multiple-echelon, multiple-commodity 
mathematical model for strategic and tactical planning. The model is developed as a MILP model in four echelons, but they did not consider satisfying the demand constraint.

Many papers have developed a supply chain network design through a mixed integer programming (MIP) model $[35,36]$. However, in fact, the quantity of the commodity is usually an integer. Our paper considers location, production, and distribution planning in the supply chain network design problem with multiechelon, multiproduct, and multiperiod characteristics in which the proposed model is pure integer linear programming (PILP) model, having four echelons, multiproduct, and multiperiod demand and satisfying a demand constraint. Consideration of using PILP is intended for providing quality guarantees of optimality [37]. Moreover, its application can be used for low volume discrete manufacturing company of large equipment. In terms of multiplicity, our paper considers the most complex model in the area of integrated production and distribution planning.

\section{Problem Definition and Model Formulation}

Development of an efficient and effective supply chain is very critical to achieving good performance. Therefore, indepth analysis is needed when opening a new plant and new distribution center in the appropriate location. Aside from that, multiple products instead of a single-product need to be considered in the problem of supply chain network design and taking into account that the integer quantity in the supply chain network design is more applicable. To deal with this problem, this paper develops a pure integer linear programming (PILP) model that focuses on determining the locations of the plants and distribution centers, as well as the number of those facilities, so that customer needs are satisfied at a minimum total cost during the planning horizon.

This research focuses on the supply chain design problem with the following characteristics.

(1) The distribution network under consideration is a multiechelon and multiproduct supply chain network.

(2) Demand in each time period (yearly) is deterministic and known.

(3) The plant or DC does not need to be opened at the beginning of the planning horizon, and when one is opened, it will not be closed.

(4) Customers can receive the product from multiple DCs.

This research develops a mathematical model that helps to determine the number and locations of plants and distribution centers in a supply network and the assignment-related demand allocation in each echelon. Figure 1 depicts the system considered in this research. According to Jayaraman and Pirkul [38], the key components of supply chain modeling that should be considered by the model builder are supply chain drivers, supply chain constraints, and supply chain decision variables of the model. Supply chain drivers represent the goal setting of the model, supply chain constraints represent the limitations on the range of decision alternatives, and supply chain decision variables are the components that set limits on the range of decision outcomes.

The objective function of this model is to minimize the total cost of the system. According to Fahimnia et al. [39], the total cost in the production and distribution network naturally consists of the production cost and distribution cost. Production cost is the sum of the fixed opening cost and the variable production cost, while the distribution cost is the sum of the fixed cost of opening the distribution center cost, the variable inventory cost, and transportation cost. Therefore, the total cost in this model consists of cost to open the plant, cost to purchase and transport raw material from supplier to plant, cost to manufacture the products, cost to open the DCs, cost to transport the product from plant to DCs, inventory holding cost of each product in DCs, and cost to transport the product from DCs to customer.

The decision variables in production and distribution planning consist of the supplier stage and distribution stage. In the supplier stage, the decision variables consist of how many suppliers should be there, how many quantity and frequency of shipment from each supplier, what is the configuration of the supplier-plant distribution network, and where are the selected locations of the suppliers and plants. The decision variables in the distribution stage consist of how many distribution centers to operate, where should they be located, and inventory in the distribution center $[40,41]$. Therefore, we decide that the decision variables in this research encompass determining where the plant and distribution center will be opened, their distribution, and the production of the plant when it is opened.

The mathematical model of this research is developed based on the model of Altiparmak et al. [28]. They set up a mathematical model that considers a single-product, four echelons, a single source, and static demand. Our paper's mathematical model has multiproducts, 4 echelons, multisources, and multiperiod demand characteristics.

To meet fluctuating customer demand, the end products and the information exchange are conducted regularly through plants and distribution centers within a given production and service network. Indicators of supply chain performance such as fill rate, customer service level, associated cost, and capability of response can be obtained under different network configurations through an evaluation of the supply chain network configuration itself. Different network configurations involve different stock levels of raw materials, subassemblies and end products, distribution center locations, production policy (make-to-stock or make-to-order), production capacity (amount and flexibility), allocation rule for limited suppliers, and transportation modes.

The common multiechelon supply chain network (MSCN) problem searches for a network configuration at a minimum cost. This is a NP-hard (nondeterministic polynomial-time hard) problem that employs a mathematical programming formulation as a natural way to build an NP-hard problem, although it is not an efficient procedure. In Yeh [42], some parameters are known in advance, namely, the numbers and capacities (demand) of suppliers, plants, distribution centers (DCs) and customers, the unit transportation cost between suppliers and plants, plants 


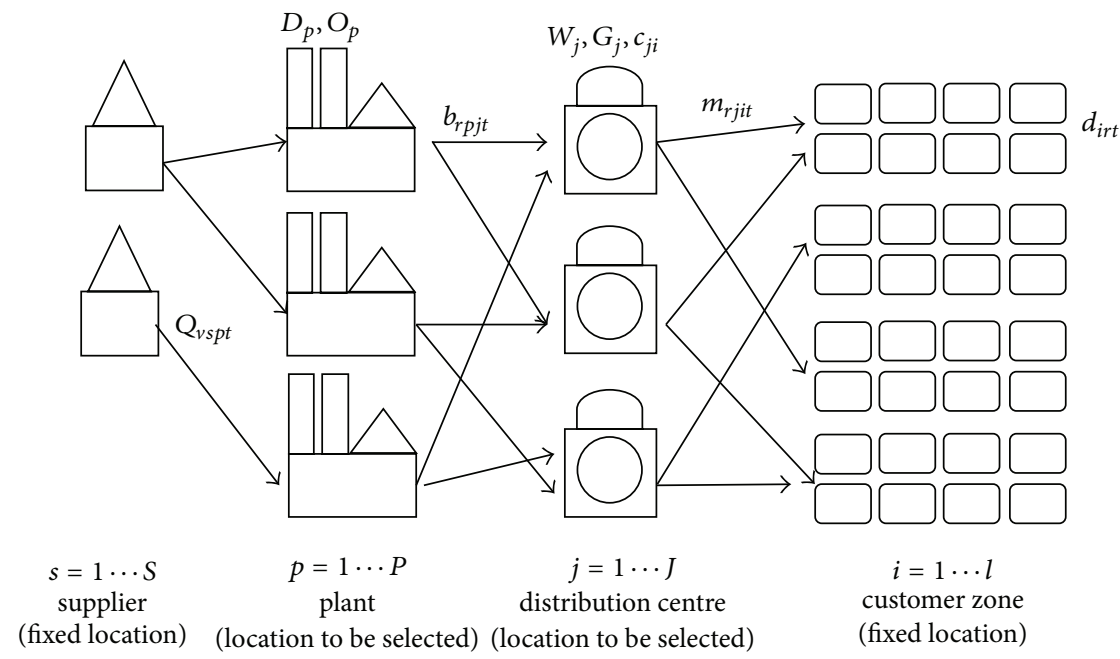

FIGURE 1: The supply chain network under consideration.

and DCs, and DCs and customers, as well as the fixed cost for operating plants and DCs. The goal of his research is to identify the locations of plants and DCs and the quantities shipped between the various points that minimize total cost and transportation costs. The problem in his research is formulated using a pure integer programming (PILP) model.

The proposed model uses the notations shown in Notations section.

The problem is formulated as follows:

$$
\begin{aligned}
\text { Min } Z= & \sum_{p}\left\{E_{p} O_{p 1}+\sum_{t=2}^{t=T} E_{p}\left(O_{p t}-O_{p(t-1)}\right)\right\} \\
& +\sum_{t=1}^{t=T} \sum_{s} \sum_{p} \sum_{v} B_{v s p} Q_{v s p t}+\sum_{t=1}^{t=T} \sum_{p} \sum_{r} A_{r p} q_{r p t} \\
& +\sum_{j}\left\{F_{j} G_{j 1}+\sum_{t=2}^{t=T} F_{j}\left(G_{j t}-G_{j(t-1)}\right)\right\} \\
& +\sum_{t=1}^{t=T} \sum_{p} \sum_{j} \sum_{r} K_{r p j} b_{r p j t} \\
& +\sum_{t=1}^{t=T} \sum_{j} \sum_{r} h_{j r t} Y_{j r}+\sum_{t=1}^{t=T} \sum_{j} \sum_{i} \sum_{r} L_{r j i} m_{r j i t},
\end{aligned}
$$

subject to

$$
\begin{aligned}
& \sum_{j} m_{r j i t} \geq d_{i r t} \quad \forall r, i, t, \\
& \sum_{p} \sum_{r} b_{r p j t} a_{r}+\sum_{r} h_{j r(t-1)} a_{r} \leq W_{j} G_{j t} \quad \forall j, t, \\
& \sum_{r} \frac{1}{\mathrm{PR}_{r}} q_{r p t} \leq D_{p} O_{p t} \quad \forall p, t,
\end{aligned}
$$

$$
\begin{aligned}
& \sum_{j} b_{r p j t} \leq q_{r p t} \quad \forall r, p, t, \\
& \sum_{r} U_{v r} q_{r p t} \leq \sum_{s} Q_{v s p t} \quad \forall v, p, t, \\
& \sum_{p} Q_{v s p t} \leq C_{s v} \quad \forall v, s, t, \\
& h_{j r t}=\sum_{p} b_{r p j t}+h_{j r(t-1)}-\sum_{i} m_{r j i t} \quad \forall j, r, t, \\
& O_{p t} \geq O_{p(t-1)} \quad \forall t=2, \ldots, T, \\
& G_{j t} \geq G_{j(t-1)} \quad \forall t=2, \ldots, T, \\
& O_{p t}=\{0,1\}, \\
& G_{j t}=\{0,1\}, \\
& Q_{v s p t} \geq 0, \text { and integer, } \\
& q_{r p t} \geq 0, \text { and integer, } \\
& b_{r p j t} \geq 0, \text { and integer, } \\
& m_{r j i t} \geq 0, \text { and integer, } \\
& h_{j r t} \geq 0, \text { and integer, } \\
& h_{j r 0}=0 \quad \forall j, r .
\end{aligned}
$$

Equation (1) shows the objective function of the model. Equation (2) is the constraint for satisfying customer demand. Equation (3) is the capacity constraint for DC $j$. Equation (4) is the capacity constraint for plant $p$. Equation (5) is the limitation of the product that is transported from plant $p$ to all DCs. Equation (6) is the requirement of raw material $v$ for production. Equation (7) is the capacity constraint for the supplier. Equation (8) is the inventory 
TABLE 1: Data for instances in the numerical experiment.

\begin{tabular}{lccc}
\hline & $\begin{array}{c}\text { Single-period } \\
\text { small-sized problem }\end{array}$ & $\begin{array}{c}\text { Multiperiod } \\
\text { small-sized problem }\end{array}$ & $\begin{array}{c}\text { Multiperiod } \\
\text { medium-sized problem }\end{array}$ \\
\hline Number of customers & 2 & 2 & 4 \\
Number of locations for distribution centers & 2 & 2 & 4 \\
Number of locations for plants & 2 & 2 & 4 \\
Number of suppliers & 2 & 2 & 4 \\
Number of products & 2 & 2 & 4 \\
Number of raw materials & 2 & 2 & 4 \\
Length of planning horizon & 1 & $2,5,10$ & 4 \\
\hline
\end{tabular}

TABLE 2: Parameter values for the numerical experiment.

\begin{tabular}{llc}
\hline NumberParameter & Generated using \\
\hline 1 & Cost to open the plant $p\left(E_{p}\right)$ & Integer uniform distribution $U(25000,30000)$ \\
2 & Maximum capacity of plant $p\left(D_{p}\right)$ & Integer $U(18,22)$ \\
3 & Production rate of manufacturing product $r\left(\mathrm{PR}_{r}\right)$ & Integer $U(10,15)$ \\
4 & Cost of transporting and purchasing raw material $v$ from supplier $s$ to plant $p\left(B_{v s p}\right)$ & $U(10,15)$ \\
5 & Unit manufacturing cost of product $r$ at plant $p\left(A_{r p}\right)$ & $U(8,10)$ \\
6 & Cost of transporting product $r$ from plant $p$ to DC $j\left(K_{r p j}\right)$ & $U(4,8)$ \\
7 & Capacity of supplier $s$ for raw material $v\left(C_{s v}\right)$ & Integer $U(1250,1500)$ \\
8 & Utilization rate of raw material $v$ per unit of finished product $r\left(U_{v r}\right)$ & Integer $U(1,5)$ \\
9 & Cost to open DC $j\left(F_{j}\right)$ & Integer $U(20000,30000)$ \\
10 & Capacity of DC $j\left(W_{j}\right)$ & Integer $U(250,350)$ \\
11 & Space requirement rate of product $r$ on a DC $\left(a_{r}\right)$ & $U(1,2)$ \\
12 & Demand at customer zone $i$ for product $r$ in time period $t\left(d_{i r t}\right)$ & Integer $U(30,50)$ \\
13 & Unit inventory holding cost of product $r$ in DC $j\left(Y_{j r}\right)$ & $U(5,10)$ \\
14 & Cost of transporting product $r$ from DC $j$ to customer $i\left(L_{r j i}\right)$ & $U(8,12)$ \\
\hline
\end{tabular}

balance equation of product $r$ in DC $j$ at time period $t$. Equation (9) ensures that the plant only opens once. Equation (10) ensures that the DC only opens once. Equations (11)-(12) are binary constraints for the decision variables. Equations (13)-(17) give the requirement of nonnegativity. Equation (18) shows the initial inventory in DC at the beginning of the planning horizon.

\section{Numerical Experiment}

This paper examines both small-sized and medium-sized problems. We conducted the experiment mainly to show the cost savings advantage of the proposed integrated model over previous related published models. Tables 1 and 2 present the indices and parameters of the model, respectively.

Table 1 gives the data of the test instances. There are five test instances: one single-period small-sized problem (Instance 1), three multiperiod small-size problems (Instances 2, 3, and 4), and one multiperiod medium-size problem (Instance 5). Instance 1 will be used as our base for the comparative analysis to show the advantages of integrating multiple period planning. In Instance 1, the numbers of customer, DC, plant, supplier, product, and raw materials are all set to be 2, except for the planning horizon which is set to be 1 year. We adopt the same data for Instances 2, 3, and 4 with different planning horizons of 2, 5, and 10 years, correspondingly. Lastly, in Instance 5, all parameters are set to be 4 .

Table 2 gives the corresponding parameter value of the model. The model consists of 14 parameters which are all generated using uniform distribution, some in integers and some in real numbers.

Our proposed model is exactly solved using LINGO. The model formulation using the LINGO framework consists of three sections: (1) sets of variables and parameters; (2) corresponding data sets; (3) mathematical model. The LINGO solver used branch and bound method to solve the problem. This method is an intelligent enumeration process seeking a sequence of better and better solutions until the best solution is found. In the process of finding the best solution, the memory is updated with the best objective function value found so far. This process continues until no further improvement can be found.

The results of location and assignment planning for the small-size numerical experiment can be seen in Tables 36. The results of location and assignment planning for the medium-size one can be seen in Table 7 .

Table 3 shows the network design results of single-period small-sized problem. This implies that having plant 2 opened materials 1 and 2 only come from supplier 2 . Plant 2 delivers all products 1 and 2 to DC 1 ; then DC 1 delivers all products 
TABLE 3: Location and assignment planning for the single-period small-sized problem.

\begin{tabular}{|c|c|c|c|}
\hline \multicolumn{2}{|c|}{ Optimal solution(objective function) } & \multicolumn{2}{|c|}{$\$ 81,105.48$} \\
\hline Period & Supplier(Plant $\left.{ }^{\text {raw material }}\right)$ & Plant(DC $\left.{ }^{\text {product }}\right)$ & $\mathrm{DC}\left(\mathrm{Cus}^{\text {product }}\right)$ \\
\hline 1 & $2\left(2^{1}, 2^{2}\right)$ & $2\left(1^{1}, 1^{2}\right)$ & $1\left(1^{1}, 1^{2}, 2^{1}, 2^{2}\right)$ \\
\hline
\end{tabular}

TABLE 4: Results for multiperiod small-size problem, $T=2$ years.

\begin{tabular}{lcrr}
\hline & Optimal solution(objective function) & & $\$ 94,766.42$ \\
Period & Supplier(Plant $\left.{ }^{\text {raw material }}\right)$ & Plant(DC $\left.^{\text {product }}\right)$ & DC(Cus $\left.{ }^{\text {product }}\right)$ \\
\hline 1 & $2\left(2^{1}, 2^{2}\right)$ & $2\left(1^{1}, 1^{2}\right)$ & $1\left(1^{1}, 1^{2}, 2^{1}, 2^{2}\right)$ \\
2 & $2\left(2^{1}, 2^{2}\right)$ & $2\left(1^{1}, 1^{2}\right)$ & $1\left(1^{1}, 1^{2}, 2^{1}, 2^{2}\right)$ \\
\hline
\end{tabular}

TABLE 5: Network design result for multiperiod small-size problem, $T=5$.

\begin{tabular}{|c|c|c|c|}
\hline \multicolumn{2}{|c|}{ Optimal solution(objective function) } & \multicolumn{2}{|c|}{$\$ 137,372.6$} \\
\hline Period & Supplier(Plant $\left.{ }^{\text {raw material }}\right)$ & Plant( $\left(\mathrm{DC}^{\text {product }}\right)$ & $\mathrm{DC}\left(\mathrm{Cus}^{\text {product }}\right)$ \\
\hline 1 & $2\left(2^{1}, 2^{2}\right)$ & $2\left(1^{1}, 1^{2}\right)$ & $1\left(1^{1}, 1^{2}, 2^{1}, 2^{2}\right)$ \\
\hline 2 & $2\left(2^{1}, 2^{2}\right)$ & $2\left(1^{1}, 1^{2}\right)$ & $1\left(1^{1}, 1^{2}, 2^{1}, 2^{2}\right)$ \\
\hline 3 & $2\left(2^{1}, 2^{2}\right)$ & $2\left(1^{1}, 1^{2}\right)$ & $1\left(1^{1}, 1^{2}, 2^{1}, 2^{2}\right)$ \\
\hline 4 & $2\left(2^{1}, 2^{2}\right)$ & $2\left(1^{1}, 1^{2}\right)$ & $1\left(1^{1}, 1^{2}, 2^{1}, 2^{2}\right)$ \\
\hline 5 & $2\left(2^{1}, 2^{2}\right)$ & $2\left(1^{1}, 1^{2}\right)$ & $1\left(1^{1}, 1^{2}, 2^{1}, 2^{2}\right)$ \\
\hline
\end{tabular}

TABLE 6: Network design results for multiperiod small-size problem, $T=10$.

\begin{tabular}{lccc}
\hline Period & $\begin{array}{l}\text { Optimal solution(objective function) } \\
\left.\text { Supplier(Plant }{ }^{\text {raw material }}\right)\end{array}$ & Plant $\left(\mathrm{DC}^{\text {product }}\right)$ & DC $\left(\mathrm{Cus}{ }^{\text {product }}\right)$ \\
\hline 1 & $2\left(2^{1}, 2^{2}\right)$ & $2\left(1^{1}, 1^{2}\right)$ & $1\left(1^{1}, 1^{2}, 2^{1}, 2^{2}\right)$ \\
2 & $2\left(2^{1}, 2^{2}\right)$ & $2\left(1^{1}, 1^{2}\right)$ & $1\left(1^{1}, 1^{2}, 2^{1}, 2^{2}\right)$ \\
3 & $2\left(2^{1}, 2^{2}\right)$ & $2\left(1^{1}, 1^{2}\right)$ & $1\left(1^{1}, 1^{2}, 2^{1}, 2^{2}\right)$ \\
4 & $2\left(2^{1}, 2^{2}\right)$ & $2\left(1^{1}, 1^{2}\right)$ & $1\left(1^{1}, 1^{2}, 2^{1}, 2^{2}\right)$ \\
5 & $2\left(2^{1}, 2^{2}\right)$ & $2\left(1^{1}, 1^{2}\right)$ & $1\left(1^{1}, 1^{2}, 2^{1}, 2^{2}\right)$ \\
6 & $2\left(2^{1}, 2^{2}\right)$ & $2\left(1^{1}, 1^{2}\right)$ & $1\left(1^{1}, 1^{2}, 2^{1}, 2^{2}\right)$ \\
7 & $2\left(2^{1}, 2^{2}\right)$ & $2\left(1^{1}, 1^{2}\right)$ & $1\left(1^{1}, 1^{2}, 2^{1}, 2^{2}\right)$ \\
8 & $2\left(2^{1}, 2^{2}\right)$ & $2\left(1^{1}, 1^{2}\right)$ & $1\left(1^{1}, 1^{2}, 2^{1}, 2^{2}\right)$ \\
9 & $2\left(2^{1}, 2^{2}\right)$ & $2\left(1^{1}, 1^{2}\right)$ & $1\left(1^{1}, 1^{2}, 2^{1}, 2^{2}\right)$ \\
10 & $2\left(2^{1}, 2^{2}\right)$ & $2\left(1^{1}, 1^{2}\right)$ & $1\left(1^{1}, 1^{2}, 2^{1}, 2^{2}\right)$ \\
\hline
\end{tabular}

to all customers. The same way of explanation uses for the solution configurations in the other columns and for the succeeding tables until Table 7. Plant 2 delivers all products 1 and 2 to DC 2; then DC 2 distributes these products to all customers. This gives us the minimum total cost of $\$ 81,105.48$.

Table 4 shows the optimal network design for Instance 2 $(T=2)$. The same optimal solution as in Table 3 is obtained for this instance for all periods. However, the optimal total cost is different with the value of $\$ 94,766.42$. Consequently, we observe a $17 \%$ increase in total cost by doubling $T$ to 2 years.

Tables 5 and 6 show the optimal network design for Instances 3 and 4 with $T=5$ and $T=10$, respectively. The same solution as in Table 3 is obtained for all periods in both instances, except that they have different optimal total costs. We have total cost of $\$ 137,372.6$ for Instance $3(T=5)$ and $\$ 207,537.2$ for Instance $4(T=10)$. Here, we observe a $51 \%$ increase in total cost by doubling the planning horizon to 10 years.

Table 7 shows the network design results of mediumsized problem. Only plants 1, 3, and 4 are opened. Supplier 1 delivers raw materials 2 and 3 to plant 1 , raw materials 2 and 4 to plant 3, and raw material 3 to plant 4 . Supplier 2 delivers raw materials 1 and 4 to plant 1 . Supplier 3 delivers raw material 2 to plant 1 , raw material 3 to plant 3 , and raw material 4 to plant 4 . Supplier 4 delivers raw material 1 to plant 2 and raw materials 1 and 2 to plant 4 . The same solution is obtained for all periods, except in period 3. In period 3, supplier 2 only delivers product to plants 3 and 4 . All DCs 1 , 2, 3, and 4 are opened for all periods. All period has different plant-DC solution mix. Furthermore, each DC delivers their corresponding product to all customers with different DCcustomer solution mix in period 4 . 
TABLE 7: Results for medium-size problem with $T=4$.

\begin{tabular}{|c|c|c|c|}
\hline \multicolumn{2}{|c|}{ Optimal solution(objective function) } & \multicolumn{2}{|c|}{$\$ 510,608.30$} \\
\hline Period & Supplier $\left(\right.$ Plant $\left.{ }^{\text {raw material }}\right)$ & $\operatorname{Plant}\left(\mathrm{DC}^{\text {product }}\right)$ & $\mathrm{DC}\left(\mathrm{Cus}^{\text {product }}\right)$ \\
\hline 1 & $\begin{array}{c}1\left(1^{2}, 1^{3}, 3^{2}, 3^{4}, 4^{3}\right) \\
2\left(1^{1}, 1^{4}\right) \\
3\left(1^{2}, 3^{3}, 4^{4}\right) \\
4\left(3^{1}, 4^{1}, 4^{2}\right)\end{array}$ & $\begin{array}{c}1\left(2^{4}, 3^{3}, 3^{4}, 4^{2}, 4^{4}\right) ; \\
3\left(1^{1}, 1^{3}, 2^{1}, 3^{2}\right) ; \\
4\left(1^{2}, 3^{2}, 3^{3}, 4^{2}, 4^{3}\right)\end{array}$ & $\begin{array}{c}1\left(1^{2}, 2^{3}, 3^{1}, 4^{1}, 4^{2}, 4^{3}\right) ; \\
2\left(1^{1}, 2^{1}, 3^{4}, 4^{1}, 4^{4}\right) ; \\
3\left(1^{4}, 2^{2}, 3^{2}, 4^{3}\right) ; \\
4\left(1^{3}, 2^{4}, 3^{3}, 3^{4}, 4^{2}\right)\end{array}$ \\
\hline 2 & $\begin{array}{c}1\left(1^{2}, 1^{3}, 3^{2}, 3^{4}, 4^{3}\right) \\
2\left(1^{1}, 1^{4}\right) \\
3\left(1^{2}, 3^{3}, 4^{4}\right) \\
4\left(3^{1}, 4^{1}, 4^{2}\right)\end{array}$ & $\begin{array}{l}1\left(2^{4}, 3^{3}, 3^{4}, 4^{2}, 4^{4}\right) \\
3\left(1^{1}, 1^{3}, 2^{1}, 3^{2}\right) \\
\quad 4\left(1^{2}, 3^{2}, 3^{3}, 4^{3}\right)\end{array}$ & $\begin{array}{c}1\left(1^{2}, 2^{3}, 3^{1}, 4^{1}, 4^{2}, 4^{3}\right) \\
2\left(1^{1}, 2^{1}, 3^{4}, 4^{1}, 4^{4}\right) \\
3\left(1^{4}, 2^{2}, 3^{2}, 4^{3}\right) \\
4\left(1^{3}, 2^{4}, 3^{3}, 3^{4}, 4^{2}\right)\end{array}$ \\
\hline 3 & $\begin{array}{c}1\left(1^{2}, 1^{3}, 3^{2}, 3^{4}, 4^{3}\right) ; \\
2\left(1^{1}, 1^{4}\right) ; \\
3\left(3^{3}, 4^{4}\right) ; \\
4\left(3^{1}, 4^{1}, 4^{2}\right)\end{array}$ & $\begin{array}{c}1\left(2^{4}, 3^{3}, 3^{4}, 4^{2}, 4^{4}\right) ; \\
3\left(1^{1}, 1^{3}, 2^{1}, 3^{2}\right) ; \\
4\left(1^{2}, 3^{2}, 3^{3}, 4^{2}, 4^{3}\right)\end{array}$ & $\begin{array}{c}1\left(1^{2}, 2^{3}, 3^{1}, 4^{1}, 4^{2}, 4^{3}\right) ; \\
2\left(1^{1}, 2^{1}, 3^{4}, 4^{1}, 4^{4}\right) \\
3\left(1^{4}, 2^{2}, 3^{2}, 4^{3}\right) ; \\
4\left(1^{3}, 2^{4}, 3^{3}, 3^{4}, 4^{2}\right)\end{array}$ \\
\hline 4 & $\begin{array}{c}1\left(1^{2}, 1^{3}, 3^{2}, 3^{4}, 4^{3}\right) \\
2\left(1^{1}, 1^{4}\right) \\
3\left(1^{2}, 3^{3}, 4^{4}\right) \\
4\left(3^{1}, 4^{1}, 4^{2}\right)\end{array}$ & $\begin{array}{c}1\left(1^{2}, 2^{4}, 3^{4}, 4^{2}, 4^{4}\right) \\
3\left(1^{1}, 1^{3}, 2^{1}, 3^{2}\right) \\
4\left(1^{2}, 3^{2}, 3^{3}, 4^{3}\right)\end{array}$ & $\begin{array}{c}1\left(1^{2}, 2^{3}, 3^{1}, 4^{1}, 4^{3}\right) ; \\
2\left(1^{1}, 2^{1}, 3^{4}, 4^{1}, 4^{4}\right) ; \\
3\left(1^{4}, 2^{2}, 3^{2}, 4^{3}\right) ; \\
4\left(1^{3}, 2^{4}, 3^{3}, 3^{4}, 4^{2}\right)\end{array}$ \\
\hline
\end{tabular}

TABLE 8: Network design results for medium-size problem with $T=4$.

\begin{tabular}{|c|c|c|c|c|c|c|c|c|c|c|c|}
\hline \multirow{2}{*}{ Year } & \multicolumn{10}{|c|}{ Annual cost (\$) } & \multirow{2}{*}{ Total } \\
\hline & 1 & 2 & 3 & 4 & 5 & 6 & 7 & 8 & 9 & 10 & \\
\hline Single-period & 81105 & 81105 & 81105 & 81105 & 81105 & 81105 & 81105 & 81105 & 81105 & 81105 & 811055 \\
\hline Multiperiod $(T=10)$ & 20754 & 20754 & 20754 & 20754 & 20754 & 20754 & 20754 & 20754 & 20754 & 20754 & 207537 \\
\hline Cost saving & 60352 & 60352 & 60352 & 60352 & 60352 & 60352 & 60352 & 60352 & 60352 & 60352 & 603518 \\
\hline
\end{tabular}

Table 8 shows the potential cost savings for multipleperiod plan versus single-period plan. For example, given the optimal cost, we have for Instance 1 a total of $\$ 811055$ which is required for a plan done individually at the beginning of each year. On the other hand, given the optimal cost of $\$ 207,537.2$ for Instance 4, we can roughly estimate average annual cost for the entire planning horizon by dividing total cost by 10 years. The potential estimated total saving is $\$ 603,518$.

It should be noted that we intend to generate a fairly concentrated demand across periods. In this way, there will be no much effect to the optimal network design across the planning horizon. With this, the results imply that the cost increases with the increases in planning horizon but not linearly as opposed to single-year planning. The fixed cost element of the total cost is distributed over the number of periods (years) included in the plan. As this number increases, this fixed cost will be stretched over the years. Thus, it ultimately gives us annual savings compared to single-year plans.

Tables 9, 10, and 11 show the production-distribution plan for Instances 1, 2, and 5, respectively. In these tables, the following optimal values are indicated, namely, production quantities needed for plants to manufacture to fully fulfill customer demand, raw materials requirements from suppliers, finished product quantities to be transferred from plant to DC, and finished product quantities to be delivered from DC to customers.
TABLE 9: Production-distribution plan for the single-period smallsized problem.

\begin{tabular}{lccc}
\hline Origin & Destination & \multicolumn{3}{c}{ Period 1 } \\
& & 1 & 2 \\
\hline Supplier 2 & Plant 2 & 521 & 445 \\
Plant 2 & DC1 & 86 & 91 \\
DC1 & Customer 1 & 44 & 45 \\
& Customer 2 & 42 & 46 \\
\hline
\end{tabular}

TABle 10: Production-distribution plan for the multiperiod smallsized problem, $T=2$.

\begin{tabular}{lccccc}
\hline \multirow{2}{*}{ Origin } & \multirow{2}{*}{ Destination } & \multicolumn{2}{c}{ Period 1 } & \multicolumn{2}{c}{ Period 2 } \\
& & 1 & 2 & 1 & 2 \\
\hline Supplier 2 & Plant 2 & 521 & 445 & 423 & 398 \\
Plant 2 & DC1 & 86 & 91 & 67 & 88 \\
DC1 & Customer 1 & 44 & 45 & 31 & 46 \\
& Customer 2 & 42 & 46 & 36 & 42 \\
\hline
\end{tabular}

\section{Sensitivity Analysis}

Jakeman et al. [27] noted that sensitivity analysis is one step in developing a model. Sensitivity analysis can also assist in executing the model [40]. Sensitivity analysis looks 
TABLE 11: Production-distribution plan for the multiperiod medium-sized problem.

\begin{tabular}{|c|c|c|c|c|c|c|c|c|c|c|c|c|c|c|c|c|c|}
\hline \multirow{2}{*}{ Origin } & \multirow{2}{*}{ Destination } & \multicolumn{4}{|c|}{ Period 1} & \multicolumn{4}{|c|}{ Period 2} & \multicolumn{4}{|c|}{ Period 3} & \multicolumn{4}{|c|}{ Period 4} \\
\hline & & 1 & 2 & 3 & 4 & 1 & 2 & 3 & 4 & 1 & 2 & 3 & 4 & 1 & 2 & 3 & 4 \\
\hline \multicolumn{18}{|c|}{ Supplier } \\
\hline \multirow{4}{*}{1} & Plant 1 & & 190 & 327 & & & 219 & 342 & & & 202 & 311 & & & 159 & 339 & \\
\hline & Plant 2 & & & & & & & & & & & & & & & & \\
\hline & Plant 3 & & 1195 & & 323 & & 1166 & & 334 & & 1182 & & 318 & & 1226 & & 274 \\
\hline & Plant 4 & & & 422 & & & & 450 & & & & 437 & & & & 450 & \\
\hline \multirow[t]{2}{*}{2} & Plant 1 & 536 & & & 818 & 583 & & & 853 & 498 & & & 758 & 645 & & & 867 \\
\hline & Plant 1 & & 12 & & & & 14 & & & & & & & & 108 & & \\
\hline \multirow[t]{2}{*}{3} & Plant 3 & & & 543 & & & & 510 & & & & 518 & & & & 540 & \\
\hline & Plant 4 & & & & 434 & & & & 414 & & & & 423 & & & & 414 \\
\hline \multirow{2}{*}{4} & Plant 3 & 393 & & & & 418 & & & & 386 & & & & 298 & & & \\
\hline & Plant 4 & 654 & 850 & & & 612 & 882 & & & 631 & 867 & & & 612 & 882 & & \\
\hline \multicolumn{18}{|l|}{ Plant } \\
\hline \multirow{4}{*}{1} & $\mathrm{DC} 1$ & & & & & & & & & & & & & & 7 & & \\
\hline & $\mathrm{DC} 2$ & & & & 41 & & & & 49 & & & & 35 & & & & 44 \\
\hline & DC3 & & & 1 & 46 & & & 2 & 34 & & & 4 & 44 & & & & 35 \\
\hline & DC4 & & 14 & & 68 & & 22 & & 74 & & 13 & & 64 & & 32 & & 71 \\
\hline \multirow{4}{*}{3} & $\mathrm{DC} 1$ & 39 & & 72 & & 55 & & 52 & & 50 & & 52 & & 74 & & 52 & \\
\hline & DC2 & 107 & & & & 101 & & & & 114 & & & & 112 & & & \\
\hline & DC3 & & 35 & & & & 42 & & & & & 34 & & & 12 & & \\
\hline & DC4 & & & & & & & & & & & & & & & & \\
\hline \multirow{3}{*}{4} & DC1 & & 47 & & & & 59 & & & & 63 & & & & 37 & & \\
\hline & DC3 & & 46 & 24 & & & 40 & 33 & & & 26 & 26 & & & 62 & 40 & \\
\hline & DC4 & & 17 & 80 & & & & 84 & & & 15 & 85 & & & & 77 & \\
\hline \multicolumn{18}{|l|}{ DC } \\
\hline \multirow{4}{*}{1} & Cust. 1 & & 45 & & & & 36 & & & & 42 & & & & 44 & & \\
\hline & Cust. 2 & & & 48 & & & & 48 & & & & 38 & & & & 48 & \\
\hline & Cust. 3 & 38 & & & & 33 & & & & 35 & & & & 45 & & & \\
\hline & Cust. 4 & 1 & 2 & 24 & & 22 & 23 & 4 & & 15 & 21 & 14 & & 29 & & 4 & \\
\hline \multirow{4}{*}{2} & Cust. 1 & 37 & & & & 36 & & & & 44 & & & & 48 & & & \\
\hline & Cust. 2 & 31 & & & & 48 & & & & 48 & & & & 46 & & & \\
\hline & Cust. 3 & & & & 1 & & & & 16 & & & & 3 & & & & 5 \\
\hline & Cust. 4 & 39 & & & 40 & 17 & & & 33 & 22 & & & 32 & 18 & 39 & & \\
\hline \multirow{4}{*}{3} & Cust. 1 & & & & 46 & & & & 34 & & & & 44 & & & & 35 \\
\hline & Cust. 2 & & 49 & & & & 48 & & & & 30 & & & & 39 & & \\
\hline & Cust. 3 & & 32 & & & & 34 & & & & 30 & & & & 35 & & \\
\hline & Cust. 4 & & & 25 & & & & 35 & & & & 30 & & & & 40 & \\
\hline & Cust. 1 & & & 30 & & & & 46 & & & & 48 & & & & 33 & \\
\hline 4 & Cust. 2 & & & & 38 & & & & 46 & & & & 37 & & & & 46 \\
\hline & Cust. 3 & & & 50 & 30 & & & 38 & 28 & & & 37 & 27 & & & 44 & 25 \\
\hline & Cust. 4 & & 31 & & & & & 22 & & & 28 & & & & 32 & & \\
\hline
\end{tabular}

at the influence of parameters changes upon the objective function. In addition, sensitivity analysis can characterize the uncertainty in the parameter [12].

We therefore conduct sensitivity analysis to analyze the changes in the decision variables and the objective function when the parameter values are changed. This section investigates the changes in decision variables, namely, $O_{p t}, Q_{v s p t}$, $b_{r p j t}, G_{j t}$, and $m_{r j i t}$, the changes in the network configuration, and the changes in objective function. We use Instance 2 to conduct the sensitivity analysis.

We performed the one-at-a-time (OAT) method in this analysis. In this method, we commonly used one parameter to change at a time while leaving all others at their baseline values. This method of sensitivity analysis considers the parameter's variability and its associated influence in the output model [43]. Table 12 presents the scenarios for 


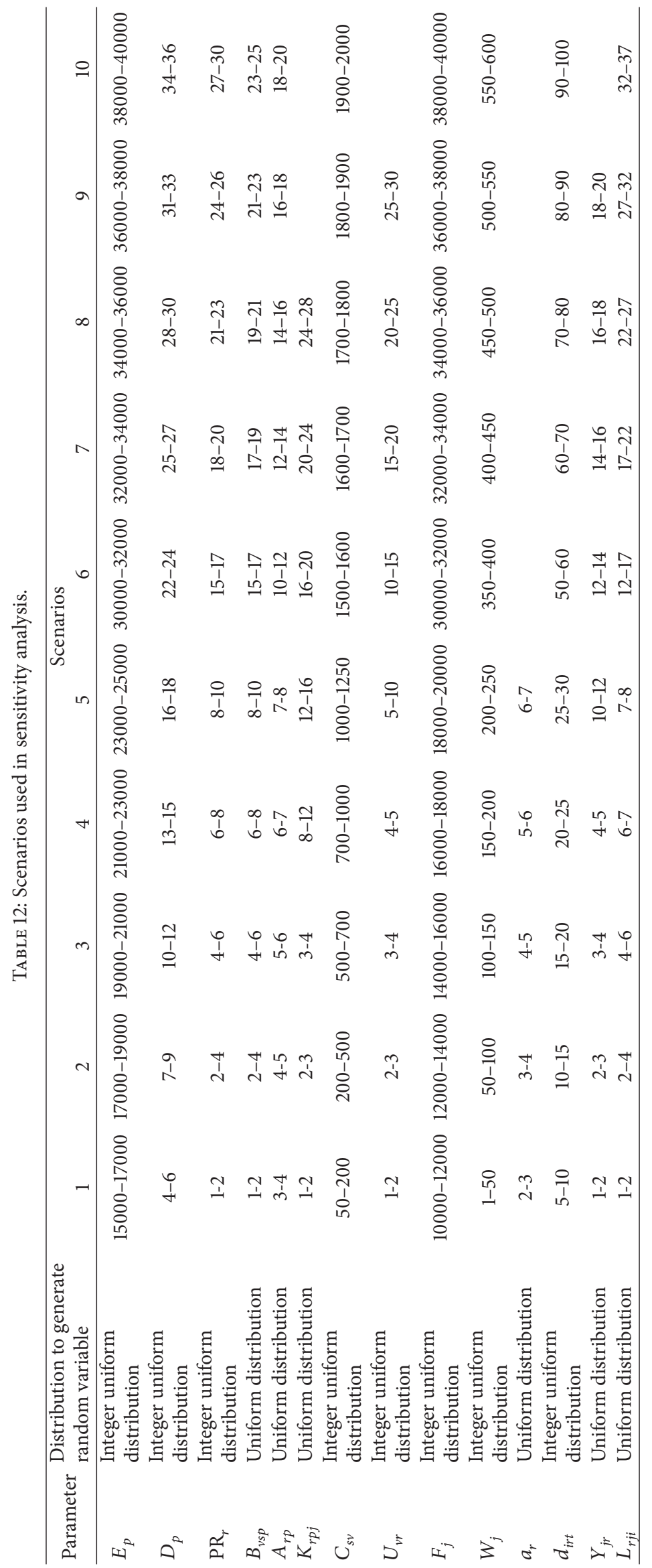


TABLE 13: Result of sensitivity analysis.

\begin{tabular}{|c|c|c|c|c|c|c|c|}
\hline \multirow{2}{*}{ Number } & \multirow{2}{*}{ Parameter } & \multicolumn{6}{|c|}{ Impact } \\
\hline & & $Z$ & $O_{p t}$ & $G_{j t}$ & $Q_{\nu s p t}$ & $b_{r p j t}$ & $m_{r j i t}$ \\
\hline 1 & Cost to open the plant $p\left(E_{p}\right)$ & $\sqrt{ }$ & - & - & - & - & - \\
\hline 2 & Maximum capacity of plant $p\left(D_{p}\right)$ & $\sqrt{ }$ & $\sqrt{ }$ & - & $\sqrt{ }$ & $\sqrt{ }$ & - \\
\hline 3 & Production rate of manufacturing product $r\left(\mathrm{PR}_{r}\right)$ & $\sqrt{ }$ & $\sqrt{ }$ & - & $\sqrt{ }$ & $\sqrt{ }$ & - \\
\hline 4 & Cost of transporting and purchasing raw material $v$ from supplier $s$ to plant $p\left(B_{v s p}\right)$ & $\sqrt{ }$ & $\sqrt{ }$ & - & $\sqrt{ }$ & $\sqrt{ }$ & - \\
\hline 5 & Unit manufacturing cost of product $r$ at plant $p\left(A_{r p}\right)$ & $\sqrt{ }$ & - & - & - & - & - \\
\hline 6 & Cost of transporting product $r$ from plant $p$ to DC $j\left(K_{r p j}\right)$ & $\sqrt{ }$ & - & - & - & - & - \\
\hline 7 & Capacity of supplier $s$ for raw material $v\left(C_{s v}\right)$ & $\sqrt{ }$ & $\sqrt{ }$ & - & $\sqrt{ }$ & $\sqrt{ }$ & - \\
\hline 8 & Utilization rate of raw material $v$ per unit of finished product $r\left(U_{v r}\right)$ & $\sqrt{ }$ & $\sqrt{ }$ & - & $\sqrt{ }$ & $\sqrt{ }$ & - \\
\hline 9 & Cost to open DC $j\left(F_{j}\right)$ & $\sqrt{ }$ & - & - & - & - & - \\
\hline 10 & Capacity of DC $j\left(W_{j}\right)$ & $\sqrt{ }$ & - & $\sqrt{ }$ & & $\sqrt{ }$ & $\sqrt{ }$ \\
\hline 11 & Space requirement rate of product $r$ in a DC $\left(a_{r}\right)$ & $\sqrt{ }$ & - & - & - & - & - \\
\hline 12 & Demand at customer zone $i$ for product $r$ in time period $t\left(d_{i r t}\right)$ & $\sqrt{ }$ & $\sqrt{ }$ & $\sqrt{ }$ & $\sqrt{ }$ & $\sqrt{ }$ & $\sqrt{ }$ \\
\hline 13 & Unit inventory holding cost of product $r$ in DC $j\left(Y_{j r}\right)$ & - & - & - & - & - & - \\
\hline 14 & Cost of transporting product $r$ from DC $j$ to customer $i\left(L_{r j i}\right)$ & $\sqrt{ }$ & - & - & - & - & - \\
\hline
\end{tabular}

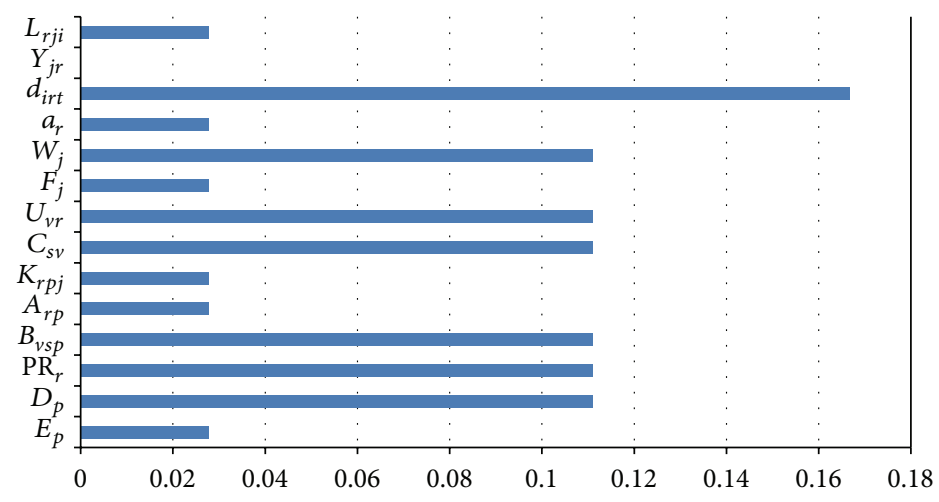

Figure 2: Percentage impact of each parameter.

sensitivity analysis. The ranges for all parameters values are given using the indicated distribution.

Table 13 shows the results of the sensitivity analysis. The impact for each parameter change is indicated with check marks under the corresponding columns for the objective function and decision variables. Changes in inventory holding cost show no impact to objective function and any of the decision variables. Parameters with the lowest impact are opening plant cost, manufacturing cost, transportation cost to $\mathrm{DC}$, opening $\mathrm{DC}$ cost, space requirement rate in $\mathrm{DC}$, and transportation cost to customer. Parameters with the medium impact are plant capacity, production rate, transportation cost to plant, supplier capacity, raw material utilization rate, and DC capacity. Finally, customer demand gives the highest impact.

In addition, the impact of the variation parameter in the objective function and decision variable are calculated as the weight of the influence, whereby the higher the value, the greater impact to the decision variables. It is calculated by dividing the number of check marks (impact) in objective function and decision variables in each parameter by total impact. The total weight of all parameters is 1 . The lowest, medium, and highest impact weights are $0.03,0.11$, and 0.17 , respectively.

The impact of each parameter to the decision variable is significantly different. It is depicted in Figure 2. Clearly, customer demand gives the greatest impact on the model's solution. Moreover, significant impact to the decision variables such as those under distribution network is also revealed. The managerial implication from this result is that the network configuration that the long-term plan may provide is an important decision-making input.

\section{Conclusions and Recommendations}

We developed an integrated model for the problem of location, production, and distribution of multiproduct, fourechelon, and multiperiod supply chain network design. The model is coded using LINGO program and implemented it for the small-sized and medium-sized problems.

The numerical experiment illustrates the applicability of the proposed model. With up to four echelons included in the 
supply chain and as much as ten years of planning horizon, we demonstrate cost savings advantage for the proposed model. Lastly, we determine the impact of all parameters involved. Customer demand gives the greatest impact on the model's solution.

The applicability of the proposed model arises, for example, mainly on manufacturing industries such as automobile, electronic, and furniture industries. We refer to the latest published models and build upon those to address some important features. However, there are some limitations of this study that may need further attention for future directions. The model belongs to the static and deterministic class with known demand. Solving this network flow problem involving four stages is computationally expensive using exact methods. Thus, we are limited with the size that the LINGO program can handle. This is when heuristic algorithms prove to be useful. For example, a trend in natureinspired algorithms such as genetic algorithm (GA) and simulated annealing (SA) to name a few is known to solve NP-hard discrete combinatorial optimization problems with high quality at faster computation speeds.

\section{Notations}

Indices
$I$ : Set of customers $(i \in I)$
$J$ : Set of potential locations of distribution centers $(j \in$ $J)$
$P$ : Set of potential locations of plants $(p \in P)$
$S$ : Set of suppliers $(s \in S)$
$R$ : Set of products $(r \in R)$
$V$ : Set of raw materials $(v \in V)$
$T$ : Length of planning horizon ( $T$ years).

Notations Corresponding to the Activities in the Plant

\section{Parameters}

$E_{p}:$ Cost to open the plant $p(\$)$

$D_{p}$ : Maximum capacity of plant $p$ (time unit)

$B_{v s p}$ : Unit cost of transporting and purchasing raw material $v$ from supplier $s$ to plant $p$ (\$/unit of raw material)

$A_{r p}$ : Unit manufacturing cost of product $r$ at plant $p$ (\$/product)

$\mathrm{PR}_{r}$ : Production rate of manufacturing product $r$ (product/time unit)

$K_{r p j}$ : Cost of transporting product $r$ from plant $p$ to DC $j$ (\$/product)

$C_{s v}$ : Capacity of supplier $s$ for raw material $v$ (raw material unit)

$U_{v r}$ : Utilization rate of raw material $v$ per unit of finished product $r$ (raw material unit/product).
Variables

$O_{p t}: 1$ if plant $p$ is opened in time period $t ; 0$ otherwise

$Q_{v s p t}$ : Quantity of raw material $v$ shipped from supplier $s$ to plant $p$ in time period $t$ (raw material unit)

$q_{r p t}$ : Quantity of product $r$ produced by plant $p$ in time period $t$ (product)

$b_{r p j t}$ : Quantity of product $r$ shipped from plant $p$ to DC $j$ in time period $t$ (product).

Notations Corresponding to the Activities in DCs

\section{Parameters}

$F_{j}$ : Cost to open DC $j(\$)$

$W_{j}$ : Capacity of DC $j$ (volume)

$a_{r}$ : Space requirement rate of product $r$ in DC (volume/product)

$d_{i r t}$ : Demand at customer zone $i$ for product $r$ in time period $t$ (product)

$Y_{j r}$ : Unit inventory holding cost of product $r$ in DC $j$ (\$/product)

$L_{r j i}$ : Cost of transporting product $r$ from DC $j$ to customer $i$ (\$/product).

Variables

$G_{j t}: 1$ if DC $j$ is opened at time period $t ; 0$ otherwise

$m_{r j i t}$ : Quantity of product $r$ shipped from DC $j$ to customer $i$ in time period $t$ (product)

$h_{j r t}$ : Quantity of product $r$ in DC $j$ at the end of time period $t$ (product).

\section{Conflict of Interests}

The authors declare that there is no conflict of interests regarding the publication of this paper.

\section{Acknowledgments}

The authors thank the editors and referees for their helpful comments and suggestions. This work was partially supported by the National Science Council of Taiwan under Grant NSC 102-2221-E-011-082-MY3. This support is gratefully acknowledged.

\section{References}

[1] J. Xu, Y. He, and M. Gen, "A class of random fuzzy programming and its application to supply chain design," Computers and Industrial Engineering, vol. 56, no. 3, pp. 937-950, 2009.

[2] Z. Tang, M. Goetschalckx, and L. McGinnis, "Modeling-based design of strategic supply chain networks for aircraft manufacturing," Procedia Computer Science, vol. 16, pp. 611-620, 2013.

[3] D. J. Thomas and P. M. Griffin, "Coordinated supply chain management," European Journal of Operational Research, vol. 94, no. 1, pp. 1-15, 1996. 
[4] A. Amiri, "Designing a distribution network in a supply chain system: formulation and efficient solution procedure," European Journal of Operational Research, vol. 171, no. 2, pp. 567-576, 2006.

[5] M. T. Melo, S. Nickel, and F. Saldanha-da-Gama, "Facility location and supply chain management-a review," European Journal of Operational Research, vol. 196, no. 2, pp. 401-412, 2009.

[6] P. N. Thanh, N. Bostel, and O. Péton, "A dynamic model for facility location in the design of complex supply chains," International Journal of Production Economics, vol. 113, no. 2, pp. 678-693, 2008

[7] F. Altiparmak, M. Gen, L. Lin, and I. Karaoglan, "A steadystate genetic algorithm for multi-product supply chain network design," Computers \& Industrial Engineering, vol. 56, no. 2, pp. 521-537, 2009.

[8] R. H. Ballou, "Unresolve issues in supply chain network design," Information Systems Frontiers, vol. 3, no. 4, pp. 417-426, 2001.

[9] M. T. Melo, S. Nickel, and F. S. D. Sa Gama, "Dynamic multicommodity capacitated facility location: a mathematical modeling framework for strategic supply chain planning," Computers \& Operations Research, vol. 33, no. 1, pp. 181-208, 2006.

[10] H. Min and G. Zhou, "Supply chain modeling: past, present and future," Computers \& Industrial Engineering, vol. 43, no. 1-2, pp. 231-249, 2002.

[11] E. H. Sabri and B. M. Beamon, "A multi-objective approach to simultaneous strategic and operational planning in supply chain design," Omega, vol. 28, no. 5, pp. 582-598, 2000.

[12] Y. Zhan and M. Zhang, "Application of a combined sensitivity analysis approach on a pesticide environmental risk indicator," Environmental Modelling \& Software, vol. 49, pp. 129-140, 2013.

[13] B. M. Beamon, "Supply chain design and analysis: models and methods," International Journal of Production Economics, vol. 55, no. 3, pp. 281-294, 1998.

[14] Z. M. Mohamed, "An integrated production-distribution model for a multi-national company operating under varying exchange rates," Internationl Journal of Production Research, vol. 58, no. 1, pp. 81-92, 1999.

[15] B. Avittathur, J. Shah, and O. K. Gupta, "Distribution centre location modelling for differential sales tax structure," European Journal of Operational Research, vol. 162, no. 1, pp. 191-205, 2005.

[16] F. Barahona and D. Jensen, "Plant location with minimum inventory," Mathematical Programming, Series B, vol. 83, no. 1-3, pp. 101-111, 1998.

[17] A. Dasci and V. Verter, "Continuous model for productiondistribution system design," European Journal of Operational Research, vol. 129, no. 2, pp. 287-298, 2001.

[18] A. Marín and B. Pelegrín, "The return plant location problem: modelling and resolution," European Journal of Operational Research, vol. 104, no. 2, pp. 375-392, 1998.

[19] S. Melkote and M. S. Daskin, "Capacitated facility location/network design problems," European Journal of Operational Research, vol. 129, no. 3, pp. 481-495, 2001.

[20] Z. M. Shen, "A profit-maximizing supply chain network design model with demand choice flexibility," Operations Research Letters, vol. 34, no. 6, pp. 673-682, 2006.

[21] K. Sourirajan, L. Ozsen, and R. Uzsoy, "A single-product network design model with lead time and safety stock considerations," IIE Transactions, vol. 39, no. 5, pp. 411-424, 2007.

[22] C.-P. Teo and J. Shu, "Warehouse-retailer network design problem," Operations Research, vol. 52, no. 3, pp. 396-408, 2004.
[23] D. Tuzun and L. I. Burke, "Two-phase tabu search approach to the location routing problem," European Journal of Operational Research, vol. 116, no. 1, pp. 87-99, 1999.

[24] Q. Wang, R. Batta, J. Bhadury, and C. M. Rump, "Budget constrained location problem with opening and closing of facilities," Computers \& Operations Research, vol. 30, no. 13, pp. 2047-2069, 2003.

[25] T.-H. Wu, C. Low, and J.-W. Bai, "Heuristic solutions to multidepot location-routing problems," Computers \& Operations Research, vol. 29, no. 10, pp. 1393-1415, 2002.

[26] A. Syarif, Y. Yun, and M. Gen, "Study on multi-stage logistic chain network: a spanning tree-based genetic algorithm approach," Computers \& Industrial Engineering, vol. 43, no. 1-2, pp. 299-314, 2002.

[27] A. J. Jakeman, R. A. Letcher, and J. P. Norton, "Ten iterative steps in development and evaluation of environmental models," Environmental Modelling \& Software, vol. 21, no. 5, pp. 602-614, 2006.

[28] F. Altiparmak, M. Gen, L. Lin, and T. Paksoy, "A genetic algorithm approach for multi-objective optimization of supply chain networks," Computers and Industrial Engineering, vol. 51, no. 1, pp. 196-215, 2006.

[29] C. Ying-Hua, "Adopting co-evolution and constraint-satisfaction concept on genetic algorithms to solve supply chain network design problems," Expert Systems with Applications, vol. 37, no. 10, pp. 6919-6930, 2010.

[30] K. S. Bhutta, F. Huq, G. Frazier, and Z. Mohamed, "An integrated location, production, distribution and investment model for a multinational corporation," International Journal of Production Economics, vol. 86, no. 3, pp. 201-216, 2003.

[31] M. E. Cóccola, M. Zamarripa, C. A. Méndez, and A. Espuña, "Toward integrated production and distribution management in multi-echelon supply chains," Computers and Chemical Engineering, vol. 57, pp. 78-94, 2013.

[32] B. Fahimnia, L. Luong, and R. Marian, "Genetic algorithm optimisation of an integrated aggregate production-distribution plan in supply chains," International Journal of Production Research, vol. 50, no. 1, pp. 81-96, 2012.

[33] M. Bashiri, H. Badri, and J. Talebi, "A new approach to tactical and strategic planning in production-distribution networks," Applied Mathematical Modelling, vol. 36, no. 4, pp. 1703-1717, 2012.

[34] H. Badri, M. Bashiri, and T. H. Hejazi, "Integrated strategic and tactical planning in a supply chain network design with a heuristic solution method," Computers and Operations Research, vol. 40, no. 4, pp. 1143-1154, 2013.

[35] B. Fahimnia, R. Z. Farahani, R. Marian, and L. Luong, "A review and critique on integrated production-distribution planning models and techniques," Journal of Manufacturing Systems, vol. 32, no. 1, pp. 1-19, 2013.

[36] C. J. Vidal and M. Goetschalckx, "Strategic productiondistribution models: a critical review with emphasis on global supply chain models," European Journal of Operational Research, vol. 98, no. 1, pp. 1-18, 1997.

[37] N. D. Crossman and B. A. Bryan, "Systematic landscape restoration using integer programming," Biological Conservation, vol. 128, no. 3, pp. 369-383, 2006.

[38] V. Jayaraman and H. Pirkul, "Planning and coordination of production and distribution facilities for multiple commodities," European Journal of Operational Research, vol. 133, no. 2, pp. 394-408, 2001. 
[39] B. Fahimnia, L. H. S. Luong, and R. M. Marian, "Optimization/ simulation modeling of the integrated production-distribution plan: an innovative survey," WSEAS Transactions on Business and Economics, vol. 5, no. 3, pp. 44-57, 2008.

[40] A. Saltelli, S. Tarantola, and F. Campolongo, "Sensitivity analysis as an ingredient of modeling," Statistical Science, vol. 15, no. 4, pp. 377-395, 2000.

[41] Ş. S. Erengüç, N. C. Simpson, and A. J. Vakharia, "Integrated production/distribution planning in supply chains: an invited review," European Journal of Operational Research, vol. 115, no. 2, pp. 219-236, 1999.

[42] W.-C. Yeh, "A hybrid heuristic algorithm for the multistage supply chain network problem," International Journal of Advanced Manufacturing Technology, vol. 26, no. 5-6, pp. 675-685, 2005.

[43] D. M. Hamby, "A comparison of sensitivity analysis techniques," Health Physics, vol. 68, no. 2, pp. 195-204, 1995. 


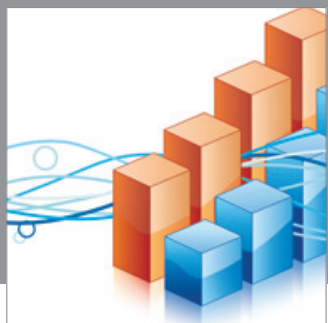

Advances in

Operations Research

mansans

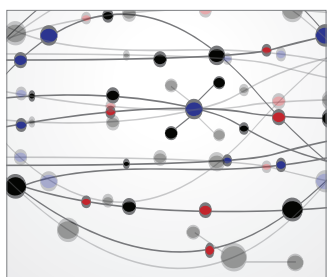

The Scientific World Journal
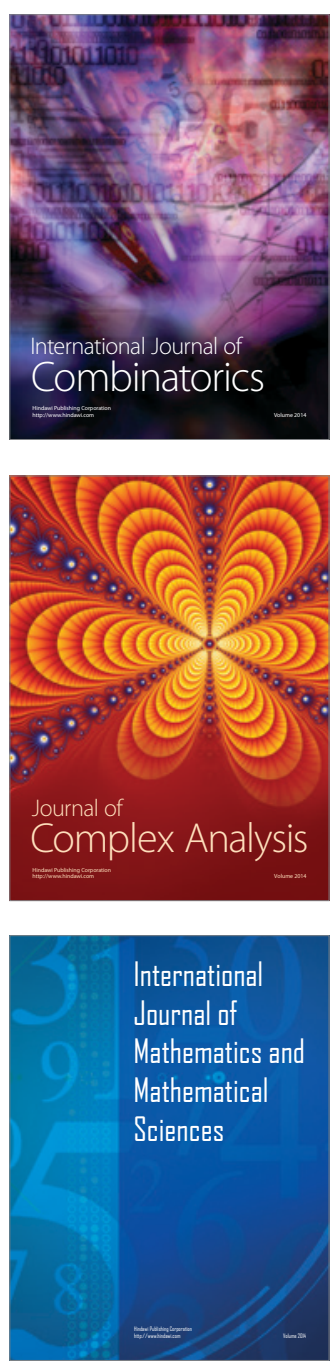
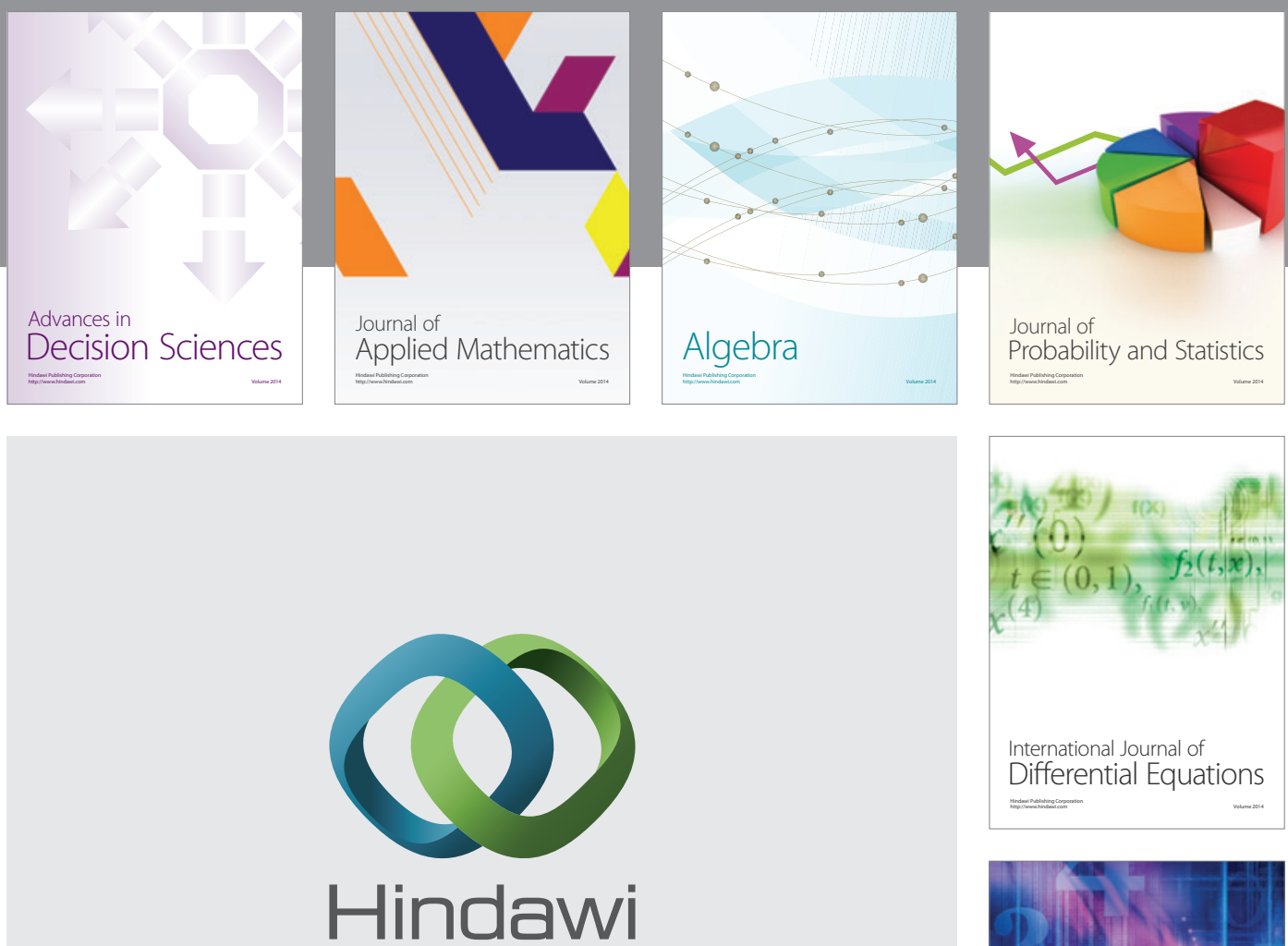

Submit your manuscripts at http://www.hindawi.com
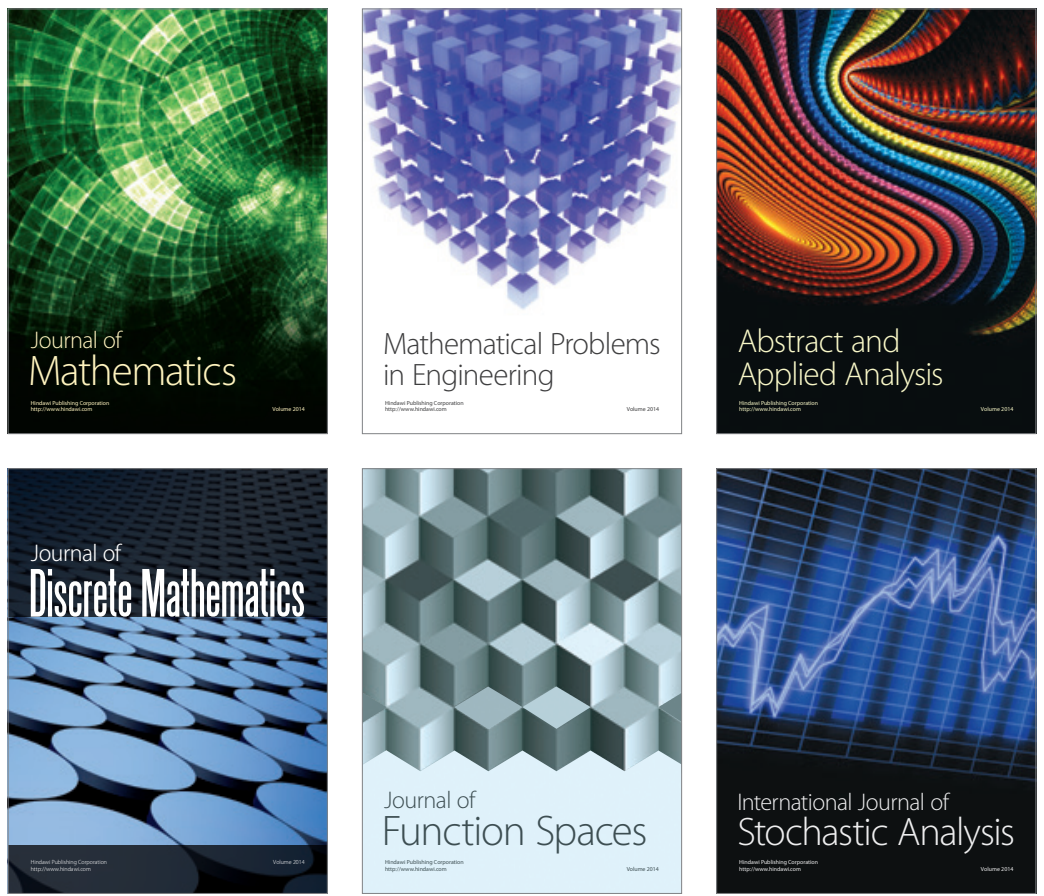

Journal of

Function Spaces

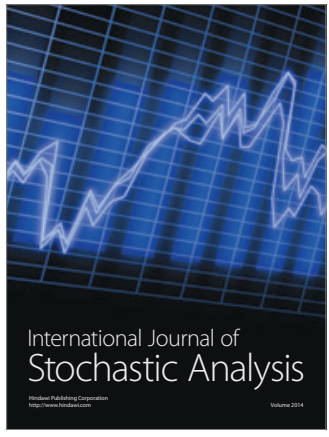

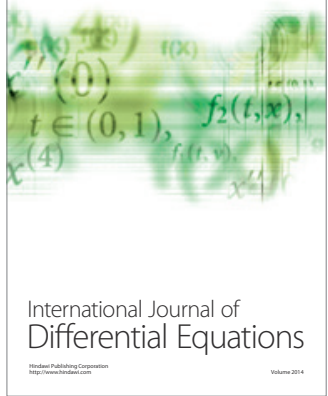
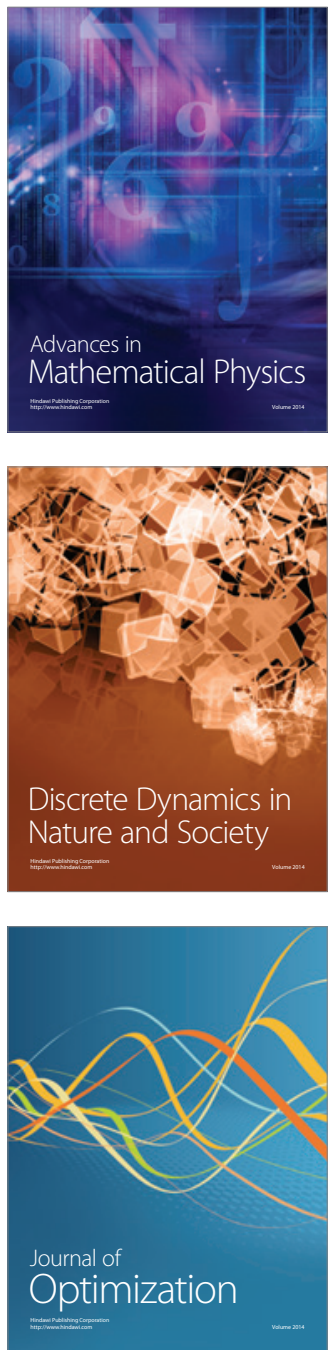